\title{
Sistema de gestión de la calidad en el hotel Brisas Covarrubias, Cuba
}

\author{
Isabel Cristina Parra García* \\ Universidad de Las Tunas. Cuba
}

Recibido: 5 de julio del 2017 / Aprobado: 24 de julio del 2017

Resumen: Se elaboró un procedimiento para el diseño del sistema de gestión de calidad (SGC) dirigido a los procesos operativos en el hotel Brisas Covarrubias, Cuba, el cual se basó en los requisitos planteados en la norma cubana (NC) ISO 9001:2015. Finalmente, se efectuó una aplicación parcial del procedimiento en el proceso operativo de Animación y se obtuvieron como resultados el mapa de procesos, el diagrama de flujo, la ficha de proceso, el documento de gestión de riesgos, el diagrama de las tendencias actuales de animación, el nivel de satisfacción del cliente (igual a $85 \%$ ), y el índice de percepción de la calidad del servicio (en 4,25).

Palabras clave: gestión de la calidad / hoteles / Cuba / satisfacción del consumidor

\section{Quality management system (OMS) in Brisas Covarrubias Hotel, Cuba}

\begin{abstract}
A procedure for the design of the SGC was elaborated directed to the operative processes in the hotel Brisas Covarrubias de Las Tunas, Cuba, based on the requirements raised in norm ISO 9001:2015. Finally a partial application of the procedure was made in the operative process of animation obtaining itself like results the map of processes, the flow chart, the card of process, the management of risks, the present tendencies of animation, the level of satisfaction of the client (equal to $85 \%$ ) and the index of perception of the quality of the service (in 4,25 ).
\end{abstract}

Keywords: quality management / hotels / Cuba / consumer satisfaction

* Correo electrónico: isabelpg@ult.edu.cu 


\section{INTRODUCCIÓN}

El turismo no es una actividad nueva en Cuba. Al igual que en el resto del mundo, tuvo su auge a inicios de la década de 1950. Sin embargo, en 1959 la economía se orientó al desarrollo de otros programas importantes en el país, debido a que en ese entonces el turismo era fundamentalmente nacional. Este hecho condicionó una estructura habitacional poco competitiva como producto internacional.

La calidad es un elemento imprescindible para mejorar los beneficios de las empresas, asegurar su competitividad y su continuidad a largo plazo dentro del mercado. Asimismo, el incremento constante de la calidad garantizará que lo que es bueno hoy seguirá siéndolo en el futuro.

Cuba no está ajena a estas tendencias mundiales. Por ello, en busca del progreso, trabaja para dar seguimiento y cumplimiento a los lineamientos de la política económica y social del partido y la revolución, en el VI Congreso del PCC. Uno de los más significativos es el número 257 del capítulo XI, Política para el Turismo, el cual plantea que se debe incrementar la competitividad de Cuba en los mercados a partir principalmente de la elevación de la calidad de los servicios y el logro de una adecuada coherencia en la relación calidad/precio.

En cuanto a los servicios turísticos, la calidad abarca los aspectos en que se entabla contacto directo con el cliente, entre los que destacan imagen del destino, promoción, acogida, información, señalización, animación, medioambiente, seguridad, coordinación y cooperación entre agentes públicos y privados.

Con el fin de lograr la calidad, es necesario gestionarla de manera correcta; para esto, existen diferentes enfoques conocidos internacionalmente. El más aplicado -sobre todo en Cuba- es el enfoque normalizado, el cual se basa en un enfoque de procesos dirigidos al cliente, la implantación de un SGC y la aplicación de los requisitos de la NC ISO 9001:2015.

Según la NC ISO 9001:2015, la adopción de un SGC es una decisión estratégica de las organizaciones; además, su diseño e implementación están influidos por los cambios y riesgos asociados al entorno externo o de sus negocios, a sus necesidades cambiantes y objetivos particulares, a los productos que proporciona, los procesos que desarrolla, y su tamaño y estructura.

En el hotel Brisas Covarrubias se encontraron un conjunto de deficiencias relacionadas con las actividades de animación ofertadas. Estas 
han dificultado el logro de los objetivos trazados por la empresa para insertarse en la industria turística y ser rentable. Luego de una observación inicial y de la revisión de documentos, se detectaron deficiencias relacionadas con estos aspectos:

- Insuficiencias en las actividades recreativas desarrolladas (las efectuadas en su mayoría son producto de improvisaciones y no se encuentran respaldadas por procedimientos y fichas técnicas).

- Poca variedad en las actividades de animación (diariamente se llevan a cabo las mismas actividades y estas no cubren todo el tiempo libre del visitante).

- El SGC en el proceso operativo de animación no cumple los requisitos de la NC ISO 9001:2015.

- No se ofrecen los servicios de buceo, que aportan ingresos.

Lo anterior permite identificar el problema de la investigación: ¿cómo mejorar la gestión de la calidad en los procesos operativos? En ese sentido, el objeto de la investigación es la gestión de la calidad en los servicios, y el objetivo general de la investigación es la elaboración de un procedimiento para el diseño del SGC dirigido a los procesos operativos, lo cual delimita como campo de acción el SGC en los procesos operativos.

Se parte de la hipótesis siguiente: la elaboración de un procedimiento para el diseño del SGC, dirigido a los procesos operativos y su aplicación parcial en el proceso de animación, beneficia la gestión de la calidad del hotel Brisas Covarrubias.

\section{Tareas específicas}

1. Sistematizar los referentes teóricos de la investigación relacionados con la gestión de la calidad en los servicios y el SGC en los procesos operativos.

2. Elaborar un procedimiento para el diseño del SGC dirigido a los procesos operativos en el hotel Brisas Covarrubias.

- Aplicar parcialmente el procedimiento para el diseño del SGC dirigido al proceso operativo de animación.

\section{MATERIALES Y MÉTODOS}

Para la llevar a cabo el procedimiento, se analizaron las diferentes bibliografías referentes al diseño del SGC. Asimismo, este procedimiento 
se elaboró en conjunto con un grupo de estudiantes de la carrera de Ingeniería Industrial.

Entre las bibliografías más importantes que han ayudado a la elaboración del procedimiento se encuentran las siguientes:

1. La metodología para un diagnóstico que permita certificar el sistema de gestión de la calidad basado en los requisitos de la NC ISO 9001:2008, desarrollada por un colectivo de autores de la Universidad de Holguín "Oscar Lucero Moya”. Esta información sirvió para la elaboración de la etapa del diagnóstico.

2. Guía de diseño para implementar el sistema de gestión de la calidad bajo la norma técnica de calidad para la gestión pública (Departamento Administrativo de la Función Pública, Red Universitaria de Extensión en Calidad, Bogotá), de los autores Luz Mary Riaño Camargo y David Hernández García (2007). Información que permitió profundizar en qué consiste la fase de diseño, su importancia, así como las diferentes etapas en que se desglosa.

3. Procedimiento para el diseño de un sistema de gestión de la calidad basado en un enfoque de procesos (tesis de grado), de los autores Eduardo Lamas Abreu y Maikel Ramos Pérez (2011). Este trabajo de investigación propició el análisis desde un enfoque en los procesos, lo cual es parte fundamental en la investigación que se llevó a cabo.

4. Guía para una gestión basada en procesos (Instituto Andaluz de Tecnología), de los autores Jaime Beltrán Sanz y Miguel Carmona Calvo. Dicha guía sirvió de base para comprender cómo trabajar enfocándose en un proceso y cómo este se puede desglosar y representar en mapas de procesos.

5. Diseño de un sistema de gestión de la calidad en el proceso de alojamiento en el hotel Gran Caribe Villa Tortuga (tesis de maestría, Universidad Tecnológica de La Habana "José Antonio Echeverría"), de las autoras Marilet Cazañas Rivero y Aleida González González (2011). Este trabajo de investigación fue la fuente de información más relevante para poder desarrollar el procedimiento, ya que se enfoca en un proceso operativo.

A continuación, se explica el procedimiento elaborado a partir de la bibliografía consultada. 


\subsection{Diagnóstico de la gestión de la calidad}

Paso 1. Análisis de la necesidad de la organización de implantar un SGC

a) El investigador debe llevar a cabo entrevistas con el encargado de atender la calidad y con el director de la organización, con el fin de corroborar que existe realmente una necesidad.

b) Creación del grupo de expertos: se determina quiénes conformarán el grupo de expertos, para lo cual se convoca al encargado de la calidad y las personas que dirigen cada proceso, entre otros que el investigador considere conveniente que desempeñen un papel clave dentro de la organización. Este grupo debe estar conformado al menos por cinco integrantes.

Paso 2. Caracterización de la organización

a) Se deben conocer brevemente la localización geográfica, misión, visión, objeto social, política y objetivos de calidad, además de cualquier otro aspecto que el investigador considere relevante. Se describirán todos estos elementos para conocer las características de la organización.

b) Caracterización del proceso operativo. En este punto se describe brevemente el proceso operativo que será objeto de investigación, también cómo se estructura la organización del proceso (organigrama) y cómo se divide; así mismo, la formación profesional del personal, cuáles son los principales clientes y proveedores.

c) Explicación del proceso. Mediante un diagrama Otida, se describe la secuencia de actividades que se desarrollan en el proceso.

d) Análisis económico de los costos del proceso. Se efectúa un breve análisis de los gastos para el proceso, tomando como muestra un periodo de tiempo (mes, trimestre, año).

Paso 3. Análisis interno y externo

En este paso se realiza un levantamiento de los principales resultados que se han obtenido hasta este punto del diagnóstico y otros elementos que el grupo de expertos consideren necesarios, para posteriormente elaborar la matriz FODA.

a) Análisis de los factores. Se escoge una guía para efectuar el análisis de los factores externos. 
b) Determinación y análisis de las no conformidades en el proceso. Se hace un registro de las principales insuficiencias detectadas en el proceso para buscar las causas que las originan, mediante un diagrama Ishikawa.

c) Encuestas a los clientes mediante el método Servqual. Se elaboran utilizando este método con sus cinco dimensiones. También se puede emplear el método Hotelqual para el caso de alojamiento, que es muy similar y se dirige específicamente a los hoteles.

d) Encuestas a los trabajadores para verificar el cumplimiento de los principios de gestión de la calidad. Se elaboran según los principios de gestión de la calidad de la familia de normas ISO 9000 vigentes, para corroborar que la organización los cumple adecuadamente.

e) Encuestas a los proveedores. Se realizan con el fin de medir si la relación de ellos con la organización es buena, regular o mala.

Paso 4. Aplicación de la lista de chequeo basado en la NC ISO 9001:2015

Paso 5. Construcción de la matriz de factores internos (MEFI), la matriz de factores externos (MEFE) y la matriz de fortalezas, oportunidades, debilidades y amenazas (FODA)

Paso 6. Propuesta de soluciones

a) Definición de las funciones del equipo de trabajo (fue constituido en el diagnóstico) para propiciar el desarrollo del procedimiento.

b) Identificación y secuenciación de los procesos (subprocesos). A partir de entrevistas con el equipo de trabajo, se pretende identificar los procesos que intervienen en la organización, así como hacer un desglose de los subprocesos que actúan dentro del proceso operativo que se esté diseñando.

- Elaboración del mapa de proceso

- Elaboración del macroproceso

c) Descripción del proceso que se diseña.

- Elaboración de los diagramas de flujos (en el caso de que se pueda).

- Confección de la ficha de proceso (son las características de los procesos).

- Identificación, evaluación y tratamiento de los riesgos de calidad por la NC ISO 31000:2009 (en proceso de actualización).

d) Identificación de las necesidades del cliente externo:

- Investigación de las nuevas tendencias del proceso. En esta subetapa se hace un estudio para conocer las tendencias que 
sigue el proceso hoy en día e identificar las preferencias de los clientes.

- Aplicación de encuestas para medir el índice de percepción de la calidad del servicio (IPCS) y el nivel de satisfacción del cliente (NSC).

Para determinar el tamaño adecuado con resultados representativos de la población, se utiliza la fórmula matemática dada por Athanassopoulus y Gounaris (2001) para calcular el tamaño de la muestra para poblaciones finita:

$$
n=\frac{N \cdot Z_{\alpha}^{2} \cdot p \cdot q}{d^{2}(N-1)+Z_{\alpha}^{2} \cdot p \cdot q}
$$

Donde:

$N=$ total de la población

$Z_{\alpha}^{2}$ : estadígrafo de la distribución normal $=1,96^{2}$

$p$ : proporción esperada (probabilidad de éxito) $=0,5$

$q$ : probabilidad de fallo $(1-p)=0,5$

$d$ : error máximo permitido que están dispuestos a cometer

$$
(d \leq 0,10)=0,05
$$

- Análisis de las principales quejas de los clientes. Se obtuvieron como resultado de las encuestas. Para este análisis, se utilizará el diagrama de Pareto.

e) Capacitación y formación de personal de la organización (esta necesidad surge de una de las fases del diagnóstico):

- Planificación de conferencias, cursos y seminarios referentes al SGC.

- Ejecución del plan de capacitación.

- Evaluación del impacto de la capacitación. Este aspecto se puede medir mediante encuestas y exámenes.

f) Documentación del SGC:

- Descripción y análisis de los puntos que componen el manual de calidad (se debe verificar que la política y objetivos de calidad se correspondan con lo establecido en NC ISO 9001:2015).

- Análisis y organización de los procedimientos, instrucciones y registros que debe contener la estructura documental del proceso.

g) Gestión de los procesos:

- Medición y seguimiento de los procesos. Se deben establecer los indicadores que permitan medir el proceso y sugerir a la organización herramientas que se consideren útiles. 
- Auditoría de los procesos. Este aspecto lo llevan a cabo los auditores internos o los que vayan a certificar el SGC.

h) Mejora de los procesos:

- Análisis de las causas que provocan las no conformidades que se obtuvieron como resultado de las auditorías a los procesos.

- Planteamiento de acciones correctivas y preventivas para minimizar las causas de las no conformidades.

- Verificación del cumplimiento de las acciones.

- Elaboración de un plan de mejora.

\section{RESULTADOS Y DISCUSIÓN}

La aplicación parcial de este procedimiento en la entidad turística que se tomó como objeto de estudio generó los siguientes resultados:

- Insuficiencias en las actividades recreativas desarrolladas, pues las ejecutadas en su mayoría son producto de improvisaciones y no se encuentran respaldadas por procedimientos ni fichas técnicas.

- Poca variedad en las actividades de animación. Diariamente se realizan las mismas actividades y estas no cubren todo el tiempo libre del visitante.

- El SGC en el proceso operativo de animación no cumple los requisitos de la norma ISO 9001:2015.

- No se ofertan los servicios de buceo que aportan ingresos de bolsillo.

La animación debe ser activa y de mejoramiento constante, por lo que el animador debe emprender algunas tareas que aseguren el éxito continuo de su trabajo. Para ello, consultará las encuestas dirigidas a los clientes con el objetivo de conocer sus intereses y poder cambiar o completar las actividades de acuerdo con sus preferencias.

La identificación de las necesidades de los clientes implicó la determinación de las características de calidad, a través de las encuestas que se les realizaron durante el periodo enero-abril del 2016.

Se utilizó el diagrama Pareto y se obtuvo lo siguiente: los medios de animación registraron un 66,7 \% de inconformidad; en el caso de los programas, la animación para niños y la animación en la playa alcanzaron $25 \%$; en cuanto al personal, el criterio predominante resultó ser el idioma con un $50 \%$. Además, se realizó el cálculo del índice de percepción de la calidad del servicio (IPCS), en el que se obtuvo 4,25, por lo que el 
NSC fue de $85 \%$. Este resultado desfavorable demostró que no estaban totalmente identificadas las necesidades de los clientes.

Tabla 1

Identificación de las características de calidad

\begin{tabular}{lccc}
\hline \multicolumn{1}{c}{ Criterios } & Incumplimientos & $\begin{array}{c}\text { Porcentaje } \\
\text { acumulado }\end{array}$ & Porcentaje \\
\hline Animación en la playa & 15 & 25 & 25 \\
Animación para niños & 15 & 50 & 25 \\
Animación diurna & 10 & 66,67 & 16,67 \\
Animación en la piscina & 10 & 83,33 & 16,67 \\
Animación nocturna & 10 & 100 & 16,67 \\
\hline Total & 60 & & \\
\hline
\end{tabular}

Elaboración propia

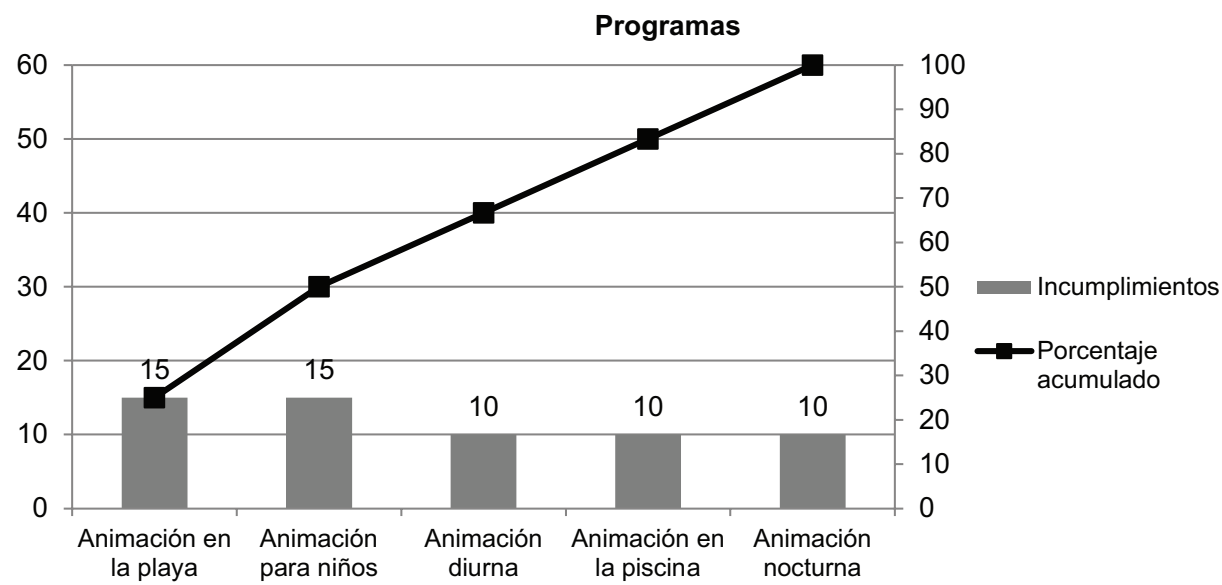

Figura 1. Diagrama de Pareto del proceso de animación Elaboración propia

Análisis de las principales quejas de los clientes:

- Aunque las actividades estaban bien distribuidas a lo largo del día, estas en su mayoría se repetían en el transcurso de la semana.

- Poca iluminación para acceder a la discoteca. 
- El show en la noche se presentaba demasiado tarde.

- Nunca estaban disponibles los servicios para las actividades náuticas, como banana, botes (porque se hallaban en mal estado o faltaba combustible). Además, había menos servicios de los publicados en la página web.

- Sería necesario mejorar la animación de formas diversas.

- No se entendía lo que decía la pizarra de animación.

- Deseo de más actividades y animación.

- Se debería brindar a los clientes un poco más de diversión deportiva y utilizar con mayor frecuencia las instalaciones deportivas.

- El gimnasio se encontraba en malas condiciones (equipamiento deteriorado e insuficiente).

- Insuficientes implementos para las actividades náuticas.

Una organización orientada a la calidad promueve una cultura que da como resultado comportamientos, actitudes, actividades y procesos para generar valor mediante el cumplimiento de las necesidades y expectativas de los clientes y otras partes interesadas pertinentes (NC ISO 9001: 2015).

En la actualidad, la competencia entre las organizaciones es cada vez más agresiva y dinámica, y suceden cambios continuos dentro de las empresas. Como resultado de estas variaciones, los clientes se vuelven más exigentes, por lo que la gestión de la calidad constituye un pilar imprescindible para el éxito de la actividad empresarial. Esto nos lleva a la necesidad de contar con un SGC que se base en las NC ISO 9001:2015 y que garantice permanentemente la calidad de los productos o servicios.

La certificación del SGC le permitiría al hotel Brisas Covarrubias aumentar su credibilidad, así como la de los servicios que presta en el mercado internacional y nacional. Esto posibilitaría, además, incrementar la eficiencia, y competitividad de la organización, debido al cumplimiento de los requisitos exigidos por el cliente y a la superación de sus expectativas. 


\section{CONCLUSIONES}

1. El procedimiento elaborado constituye una herramienta para el diseño de un sistema de gestión de la calidad que tenga en cuenta las etapas del ciclo de Deming (planificar, hacer, verificar, actuar) y puede ser aplicable en todos los procesos de la instalación.

2. Asimismo, permitió que se diseñara parcialmente el SGC en el proceso operativo de animación. Esto evidencia que el hotel continúa trabajando para la mejora continua de la calidad.

\section{RECOMENDACIONES}

1. Perfeccionar el procedimiento propuesto a partir de su validación práctica en diferentes organizaciones con características similares.

2. Capacitar al personal, no solo en materia de calidad, sino también en el ámbito estudiantil (especialización) para lograr crear una fuerza de trabajo calificada que sustente al funcionamiento eficaz del SGC.

\section{REFERENCIAS}

Almaguer De Miguel, A. (2015). Propuesta de un plan de mejora de la gestión de la calidad en el hotel Brisas Covarrubias, Puerto Padre, Las Tunas (trabajo de diploma para optar el título de ingeniero industrial). Universidad Vladimir I. Lenin. Las Tunas, Cuba.

Athanassopoulus, A., Gounaris, S., y Stathakopoulus, V. (2001). Behavioral Responses to Customer Satisfaction: An Empirical Study. European Journal of Marketing, 35(5/6), 687-707.

Camisón Zornoza, C. (2004). Estrategias de calidad turísticas. El papel de los sistemas de acreditación y certificados de calidad. Revista Mediterráneo Económico, 8(5), 118-168.

Carrasco Fernández, S. (2013). Procesos de gestión de calidad en hostelería y turismo. Madrid: Paraninfo.

Cazañas Rivero, M. (2012). Diseño e implantación del SGC en el proceso de alojamiento en el hotel Gran Caribe "Villa Tortuga" (tesis de máster). Instituto Superior Politécnico José Antonio Echeverría. La Habana, Cuba.

Grupo Cubanacán. (2008). Manual operativo hoteles Brisas. La Habana: Autor. 
Hernández-Concepción, I. (s. f.). Metodología para implementar la gestión de la calidad y la reingeniería de procesos. Experiencias prácticas. En XV Fórum Nacional de Ciencia y Técnica (Aporte nuevo). Holguín: Editorial Pueblo y Educación.

Hernández Escobar, D. (2013). Actividades de animación sociocultural para insertar en el programa de animación del Hotel Brisas Covarrubias (trabajo de diploma para licenciatura en Turismo). Universidad de Holguín, Cuba.

Medina, A. (s. f.). Tendencias hoteleras. Recuperado de http://trafficamerican.com

Oficina Nacional de Normalización. (2001). NC ISO 9004. Sistemas de gestión de la calidad. Directrices para la mejora continua del desempeño. (2001). La Habana: Autor.

Oficina Nacional de Normalización. (2008). NC ISO 9001. Sistema de gestión de la calidad - requisitos. La Habana: Autor.

Oficina Nacional de Normalización (2014). NC 127.2014. Industria turística - requisitos para la clasificación por categorías de los establecimientos de alojamiento turístico. La Habana: Autor.

Oficina Nacional de Normalización. (2015a). NC ISO 9000. Sistema de gestión de la calidad-fundamentos y vocabulario. La Habana: Autor.

Oficina Nacional de Normalización. (2015b). NC ISO 9001. Sistema de gestión de la calidad-requisitos. La Habana: Autor.

Partido Comunista de Cuba. (2011). Lineamientos de la política económica y social del partido y la revolución. En VI Congreso del PCC (p. 33). La Habana: Editora Política.

Sánchez Leyva, R. (2012). Propuesta de actividades para potenciar la animación turística en el hotel Playa Pesquero (trabajo de diploma para licenciatura en Turismo). Universidad de Holguín.

Ulacia Oviedo, Z. (2000). Selección de lecturas sobre temas de animación turística (partes I y II). La Habana: Centro de Estudios Turísticos. 